\title{
Analyze and calculation of coupling coefficient based on evanescence field for plasmonic directional coupler structure
}

\author{
Mohsen Olyaee $^{1} \cdot$ Mohammad Bagher Tavakoli $^{1} \cdot$ Abbas Mokhtari $^{1}$
}

Received: 16 November 2017 / Accepted: 15 October 2018 / Published online: 25 October 2018

(c) The Author(s) 2018

\begin{abstract}
We obtain the calculation of coupling coefficient of plasmonic directional coupler made up of two monolayer waveguides separated in the range of $200 \mathrm{~nm}$ for TM mode and we assume each waveguides acts as a perturbation to other waveguide but does not affect the waveguide mode. We simulation the transfer distance respect to normalized frequency in two directions, $x$ and $\mathrm{z}$, values transfer distance a limitation of a bandwidth is suitable for designing of integrated optical circuits and construction of couplers and switches.
\end{abstract}

Keywords Coupling coefficient $\cdot$ Plasmonic $\cdot$ Coupler

\section{Introduction}

Recent advances in the study of nanophotonics imply a fast developing and new promising field of science and technology which named plasmonics for exploiting optical features of metallic nano structures. Surface Plasmon Polaritons (SPPs) waves propagating at the interface between a metal and a dielectric. SPPs are electromagnetic waves that propagate at the surface of a metal. Their evanescent field tail typically extends several hundreds of nanometers into the surrounding dielectric. Near the surface plasmon frequency, the SPP dispersion relation deviates strongly from the light line toward larger wave vectors, thus enabling short plasmon wavelengths at optical frequencies. The dispersion relation of plasmons can be further tuned in metal-insulator-metal (MIM) geometries, in which the plasmon field is confined in a dielectric gap between two metal layers. Due to intercoupling of plasmons at both metal-dielectric interfaces, plasmon modes with symmetric and antisymmetric magnetic field distribution exist, of which the latter shows the largest dispersion and highest loss. Such material systems have shown their potential to spatially confine and manipulate light in sub wavelength scale

This article is part of the Topical Collection on Optical Wave and Waveguide Theory and Numerical Modelling.

Mohammad Bagher Tavakoli

mtb1357@yahoo.com

1 Department of Engineering, Arak Branch, Islamic Azad University, Arak, Iran 
because of highly matched SPPs a mixed wave of light and free electron oscillation of conductors, actually on the metal surface (Du and Chiou 2014). Besides the applications of plasmonic devices in medical and biological fields such as using surface-enhanced Raman spectroscopy, the amazing aspect of plasmonic devices, confining light, makes such devices to have versatile applications for designing various type of devices i.e. different components of plasmonic circuits such as waveguides, couplers, switches and so on (Pala et al. 2008; Brongersma and Kik 2007). Because of the profound penetration of field into the metals and the material loss, using plasmonic waveguides is not that good for propagation in long-range. On the other hand they are good candidate for integrated optical circuit application in small scale regimes (Maier 2007). In this research initially we obtain the coupling coefficient of plasmonic directional coupler which made up of two parallel plasmonic waveguide separated by a distance. We will use the evanescence field of two waveguides that induced polarization to obtain coupling coefficient. Transfer distance will be calculated numerically respect to normalized frequency and simulated for both components.

\section{Equations}

Monolayer plasmonic waveguide is one of the simplest waveguide that actually is an interface between a metal and dielectric (Pleros et al. 2011). In this paper, we incorporate two monolayer plasmonic waveguides which separate each other and we assume each waveguides act as a perturbation to other waveguide but do not affect the other waveguide mode. Figure 1 illustrate a directional coupler that made of two separate monolayer plasmonic metal-insulator-metal (MIM) waveguide by a distance $2 \mathrm{a}$ and filled by dielectric $\varepsilon$. The permittivity of metallic part of plasmonic waveguide is demonstrated as $\varepsilon_{c}$. While the MIM waveguides feature lower propagation lengths compared to insulator-metal-insulator (IMI) waveguides, the higher confinement results in a tighter pitch (center-to-center distance between the adjacent waveguides) while maintaining a low crosstalk noise. This is critical for signal integrity. The crosstalk noise, defined as the coupling or overlap of modes between the adjacent waveguides resulting in a transfer of power from one waveguide to another is measured in terms of transfer distance.

Monolayer plasmonic waveguide only supports TM modes because only these modes can excite Plasmon polaritons by tangential component of its electric field. If we take the coordinate system as shown in Fig. 1, the components of electric fields of TM mode

Fig. 1 Schematic of two monolayer MIM waveguides for directional coupler

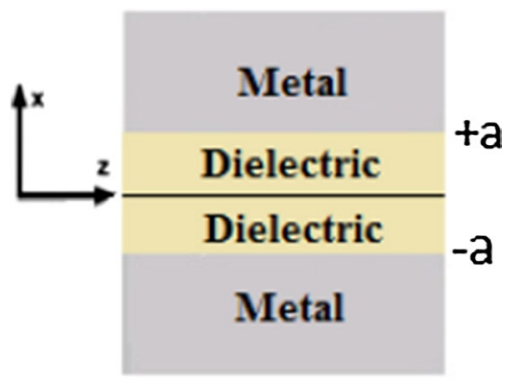


are given by $E_{x}$ and $E_{z}$. We take the upper waveguide of plasmonic directional coupler as number 1 and the lower one as number 2, in general the electric field of upper and lower waveguide of plasmonic directional coupler by taking into account a weak interaction of two waveguides are written as following:

$$
\begin{aligned}
& E_{x 1}(x, z)=A_{1}(z) F_{1}(x) e^{i \beta z} \\
& E_{z 1}(x, z)=B_{1}(z) G_{1}(x) e^{i \beta z} \\
& E_{x 2}(x, z)=A_{2}(z) F_{2}(x) e^{i \beta z} \\
& E_{z 2}(x, z)=B_{2}(z) G_{2}(x) e^{i \beta z}
\end{aligned}
$$

where $A$ and $B$ are slowly varying amplitudes, $F(x)$ and $G(x)$ are normalized transvers functions of each waveguide alone respectively. Lower waveguide undergoes a perturbation of the medium for upper waveguide and vice versa. This perturbation is appearance as difference of refractive index $n_{c}-n$. By implying Helmholtz equations to electric fields one can write:

$$
\begin{aligned}
& \nabla^{2} E_{x 1}+k_{c}^{2} E_{x 1}=-\mu_{0} \omega^{2} \varepsilon_{0}\left[\left(\varepsilon_{c}+i \frac{\sigma}{\varepsilon_{0} \omega}\right)-\varepsilon\right] E_{x 2} \\
& \nabla^{2} E_{z 1}+k_{c}^{2} E_{z 1}=-\mu_{0} \omega^{2} \varepsilon_{0}\left[\left(\varepsilon_{c}+i \frac{\sigma}{\varepsilon_{0} \omega}\right)-\varepsilon\right] E_{z 2} \\
& \nabla^{2} E_{x 2}+k_{c}^{2} E_{x 2}=-\mu_{0} \omega^{2} \varepsilon_{0}\left[\left(\varepsilon_{c}+i \frac{\sigma}{\varepsilon_{0} \omega}\right)-\varepsilon\right] E_{x 1} \\
& \nabla^{2} E_{z 2}+k_{c}^{2} E_{z 2}=-\mu_{0} \omega^{2} \varepsilon_{0}\left[\left(\varepsilon_{c}+i \frac{\sigma}{\varepsilon_{0} \omega}\right)-\varepsilon\right] E_{z 1}
\end{aligned}
$$

where $\varepsilon_{0}, \mu_{0}, \sigma, \omega$ are the vacuum electric permittivity, vacuum magnetic permeability, conductivity and frequency, respectively. $k$ is the wave vector which is written for metals by a subscript $\mathrm{c}$ and

$$
\begin{gathered}
k_{c}^{2}=\beta^{2}-k_{0}^{2} \varepsilon_{c} \\
k^{2}=\beta^{2}-k_{0}^{2} \varepsilon
\end{gathered}
$$

That $\beta$ is propagation constant written as

$$
\beta=k_{0} \sqrt{\frac{\varepsilon \varepsilon_{c}}{\varepsilon+\varepsilon_{c}}}
$$

We suppose that propagation constant and transverse field distribution is unchanged under the effect of this perturbation, only the amplitudes $A_{1}(z), A_{2}(z), B_{1}(z)$ and $B_{2}(z)$ are taken as function of $z$ although for monolayer plasmonic waveguide alone are constant. If the slowly varying amplitude is impose to equation up four coupled first-order differential equations are given:

$$
\frac{\partial A_{1}}{\partial z}=i C_{12} A_{2}
$$




$$
\begin{aligned}
& \frac{\partial B_{1}}{\partial z}=i C_{12} B_{2} \\
& \frac{\partial A_{2}}{\partial z}=i C_{21} A_{1} \\
& \frac{\partial B_{2}}{\partial z}=i C_{21} B_{1}
\end{aligned}
$$

Parameter Cs are called coupling coefficients and defined as following:

$$
\begin{aligned}
& C_{12 x}=\frac{1}{2}\left[\left(\varepsilon_{c}+i \frac{\sigma}{\varepsilon_{0} \omega}\right)-\varepsilon\right] \frac{k_{0}^{2}}{\beta} \int_{-\infty}^{-a} F_{1}^{*} F_{2} d x \\
& C_{12 z}=\frac{1}{2}\left[\left(\varepsilon_{c}+i \frac{\sigma}{\varepsilon_{0} \omega}\right)-\varepsilon\right] \frac{k_{0}^{2}}{\beta} \int_{-\infty}^{-a} G_{1}^{*} G_{2} d x \\
& C_{21 x}=\frac{1}{2}\left[\left(\varepsilon_{c}+i \frac{\sigma}{\varepsilon_{0} \omega}\right)-\varepsilon\right] \frac{k_{0}^{2}}{\beta} \int_{+a}^{+\infty} F_{1}^{*} F_{2} d x \\
& C_{12 z}=\frac{1}{2}\left[\left(\varepsilon_{c}+i \frac{\sigma}{\varepsilon_{0} \omega}\right)-\varepsilon\right] \frac{k_{0}^{2}}{\beta} \int_{+a}^{+\infty} G_{1}^{*} G_{2} d x
\end{aligned}
$$

The normalized transvers functions, $F(x)$ and $G(x)$ are written as the normalized field distribution of monolayer plasmonic waveguide (LyGagnon et al. 2012).

Some calculations lead to the four coupling coefficients as follow:

$$
\begin{gathered}
C_{12 x}=C_{21 x}=\frac{1}{2}\left[\left(\varepsilon_{c}+i \frac{\sigma}{\varepsilon_{0} \omega}\right)-\varepsilon\right] \frac{k_{0}^{2}}{\beta} \int_{-\infty}^{-a} F_{1}^{*} F_{2} d x=-i\left[\left(\varepsilon_{c}+i \frac{\sigma}{\varepsilon_{0} \omega}\right)-\varepsilon\right] \frac{k_{0}^{2}}{\beta} \frac{\sqrt{\varepsilon \varepsilon_{c}}}{\left(\varepsilon_{c-} \varepsilon\right)\left(k+k_{c}\right)} e^{-2 k a} \\
C_{12 z}=C_{21 z}=\frac{1}{2}\left[\left(\varepsilon_{c}+i \frac{\sigma}{\varepsilon_{0} \omega}\right)-\varepsilon\right] \frac{k_{0}^{2}}{\beta} \int_{-\infty}^{-a} G_{1}^{*} G_{2} d x=-i\left[\left(\varepsilon_{c}+i \frac{\sigma}{\varepsilon_{0} \omega}\right)-\varepsilon\right] \frac{k_{0}^{2}}{\beta} \frac{\sqrt{k k_{c} \varepsilon \varepsilon_{c}}}{\left(\varepsilon_{c-} \varepsilon\right)\left(k+k_{c}\right)} e^{-2 k a}
\end{gathered}
$$

Thus we have a plasmonic directional coupler which has capability to couple the optical power from one waveguide into another. The coupling length or transfer distance in both directions of $x$ and $z$ are calculated as

$$
L_{x}=\frac{\pi}{2 C_{12 x}} L_{z}=\frac{\pi}{2 C_{12 z}}
$$

We simulated the transfer distance for both components by using Au as the metals and $A s_{2} S_{3}$ chalcogenide glass for dielectric layer. The results of simulations have been shown in Figs. 2 and 3. In each figure the horizontal axis is normalize frequency respect to plasma frequency $\omega_{p}=1.36 \times 10^{16} \mathrm{~Hz}$. Figure 2 shows the $\mathrm{x}$ component of real part of transfer distance and Fig. 3 shows the $\mathrm{z}$ component of it respect to normalize frequency (de Oliveira et al. 


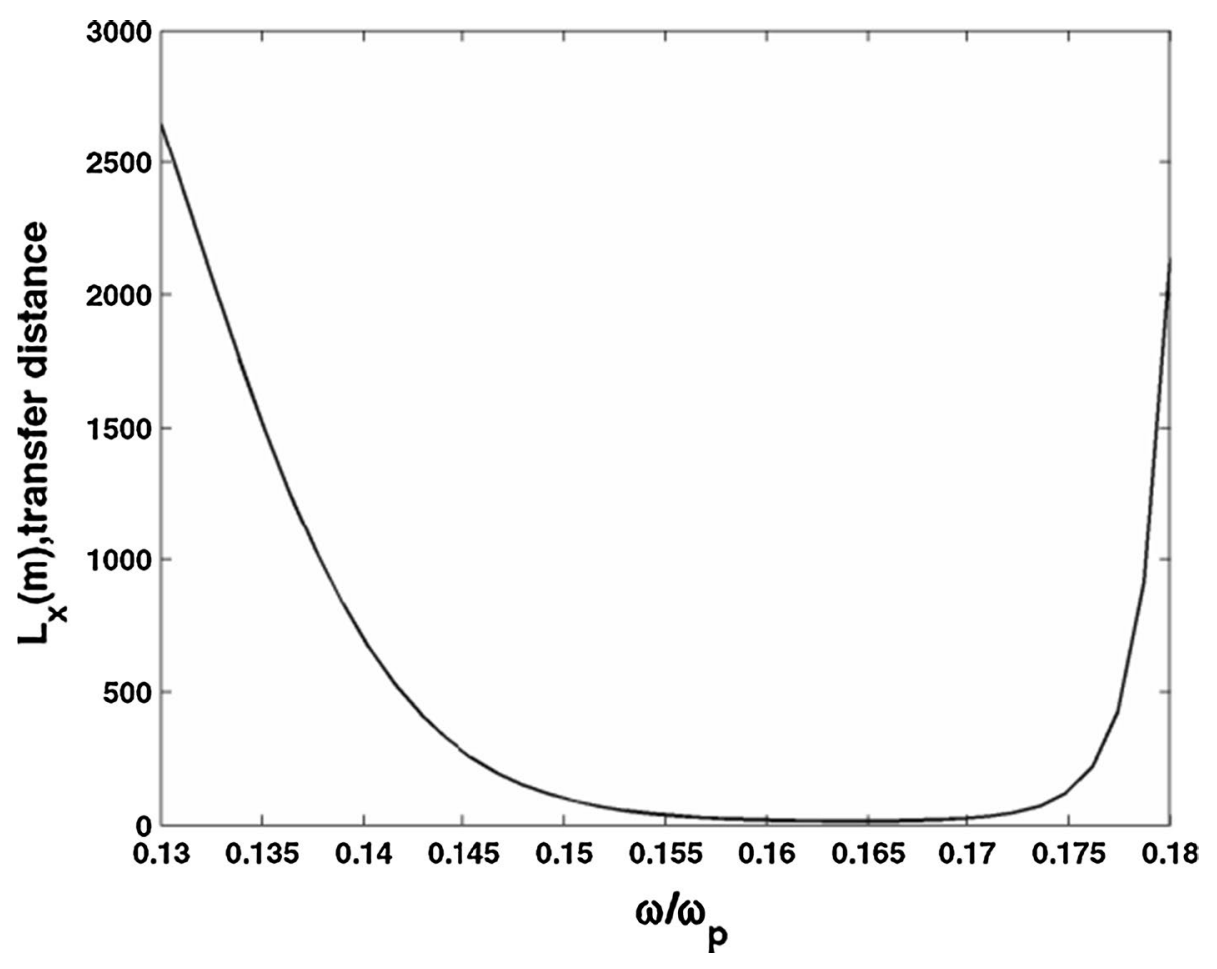

Fig. 2 Real part of transfer distance as a function of normalized frequency in direction of $\mathrm{x}$ axis for nano plasmonic directional coupler at $\mathrm{a}=100 \mathrm{~nm}$

2012). The general behavior of $L_{z}$ and $L_{x}$ are the same initially decrease by increasing the frequency and then increase and in some interval of frequency the value of two transfer lengths are nearly constant and slightly change the flat interval by increasing the distance between two monolayer waveguide. On the other hand there is a huge difference between $L_{x}$ and $L_{z}$ because of their values, the value of $L_{x}$ is in the order of kilometers while the order of $L_{z}$ is under millimeters. We expected such result because the x component of electric field has a few contributions to induce polarization or current to other waveguide. So because of weak coupling a large transfer distance of $L_{z}$, it is not useful for designing integrated optical devices. On the other hand the values of $L_{z}$ is small enough and it can be useful for designing switches or $3 \mathrm{db}$ couplers for integrated optical circuits manufacturing. The minimum value of $L_{z}$ is occurred at $\omega / \omega_{p}=0.165$ is equal to $0.66 \mu \mathrm{m}$ for $2 \mathrm{a}=200 \mathrm{~nm}$.

The interesting result is the good flatness of transfer distance in the interval between $\omega / \omega_{p}=0.15$ and $\omega / \omega_{p}=0.175$, this remarkable feature is made plasmonic directional couplers a good candidate for designing dispersion less photonic devices. 


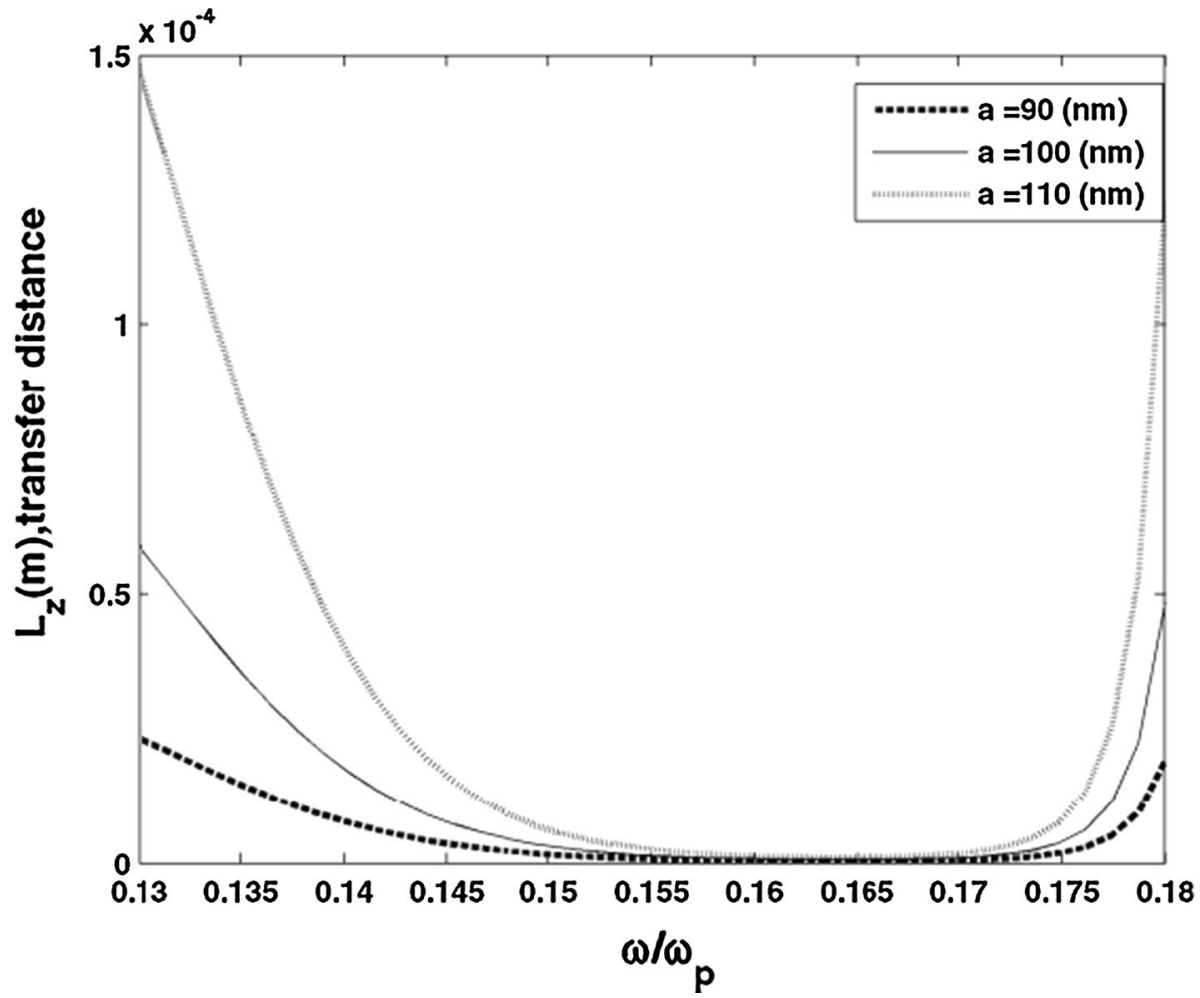

Fig. 3 Real part of transfer distance as a function of normalized frequency in direction of $\mathrm{z}$ axis for nano plasmonic directional coupler at three distances $a=90 \mathrm{~nm}, \mathrm{a}=100 \mathrm{~nm}$ and $\mathrm{a}=110 \mathrm{~nm}$

\section{Conclusion}

Confining light in plasmonic devices makes such devices to have versatile applications for designing various types of devices i.e. different components of plasmonic circuits such as waveguides, couplers, switches and so on. In this research we obtained the coupling coefficient of plasmonic directional coupler. We calculated transfer distance numerically respect to normalized frequency and simulated it for both components. The value of $L_{x}$ which related to $E_{x}$ is in the order of kilometers while the order of $L_{z}$ related to $E_{z}$ is under millimeter. So because of weak coupling a large transfer distance of $L_{z}$ it is not useful for designing integrated optical devices. On the other hand the values of $L_{z}$ is small enough and it can be useful for designing switches or $3 \mathrm{db}$ couplers for integrated optical circuits manufacturing. Also we found the optimum point of $L_{z}$ and the band width in which $L_{z}$ is almost flatness that is remarkable feature to make plasmonic directional couplers a good candidate for designing dispersion less photonic devices.

Open Access This article is distributed under the terms of the Creative Commons Attribution 4.0 International License (http://creativecommons.org/licenses/by/4.0/), which permits unrestricted use, distribution, and reproduction in any medium, provided you give appropriate credit to the original author(s) and the source, provide a link to the Creative Commons license, and indicate if changes were made. 


\section{References}

Brongersma, M.L., Kik, P.G.: Surface Plasmon Nanophotonics. Springer, Berlin (2007)

de Oliveira R.E.P., Lipson M., de Matos C.J.S.: Electrically controlled silicon nitride ring resonator for quasi-phase matched second-harmonic generation. In: CLEO: Science and Innovations. Optical Society of America (2012)

Du, C.H., Chiou, Y.P.: Vertical directional couplers with ultra-short coupling length based on hybrid plasmonic waveguides. J. Light. Technol. 32(11), 2065-2071 (2014)

LyGagnon, D.S., Balram, K.C., White, J.S., Wahl, P., Brongersma, M.L., Miller, D.A.B.: Routing and photodetection in subwavelength plasmonic slot waveguides. J. Nanophotonics 1(1), 9-16 (2012)

Maier, S.A.: Plasmonics, Fundamentals and Applications. Springer, New York (2007)

Pala, R.A., Shimizu, K.T., Melosh, N.A., Brongersma, M.L.: A nonvolatile plasmonic switch employing photochromic molecules. Nano Lett. 8, 1506-1510 (2008)

Pleros, N., Kriezis, E.E., Vyrsokinos, K.: Optical interconnects using plasmonics and Si-photonics. IEEE J. Photonics 3(2), 296-301 (2011) 